\title{
Hydroxychloroquine and visual screening in a rheumatology outpatient clinic
}

\author{
D J Grierson
}

\begin{abstract}
Objective-To review 10 years' data relating to visual screening of patients taking hydroxychloroquine.

Methods-Following baseline visual assessment, ophthalmic monitoring was carried out at six monthly intervals on 758 patients while on hydroxychloroquine. This consisted of corrected visual acuity, central field screening with a red Amsler grid, slit lamp examination, and retinoscopy.

Results-None of the patients suffered visual impairment from retinal toxicity, though 12 reported visual disturbance. This was related to ocular muscle imbalance in four. In the remainder, none of the ocular findings was directly attributable to hydroxychloroquine. Ten patients reported defects when tested with a red Amsler grid. None was related to retinal toxicity. Seven patients developed corneal drug deposits which cleared on stopping or reducing the dose of hydroxychloroquine.
\end{abstract}

Conclusions-The findings support the view that following baseline ophthalmic examination for patients receiving hydroxychloroquine, regular ophthalmic screening is not required if the daily dose is less than $6.5 \mathrm{mg} \mathrm{kg}^{-1}$ and the cumulative dose is less than $200 \mathrm{~g}$.

(Ann Rheum Dis 1997;56:188-190)

Hydroxychloroquine is now routinely used in medicine in the management of connective tissue and skin disorders. The College of Ophthalmologists published a document in $1993^{1}$ relating to the use of antimalarials and ophthalmic screening. They suggest that patients on hydroxychloroquine do not require ophthalmic monitoring provided that the daily dose of hydroxychloroquine sulphate is less than $6.5 \mathrm{mg} \mathrm{kg}^{-1}$ of lean body weight ${ }^{2}$ and the cumulative dose does not exceed 200 g. In support of this view, data are presented on patients receiving treatment with hydroxychloroquine who were referred for visual assessment to the ophthalmology department at Glasgow Royal Infirmary between 1983 and 1995.

\section{Methods}

Data were collected prospectively with regard to ocular examination and drug dosage. Patient weights were not included in the original data collection and were retrieved from the medical records at a later date (actual rather than ideal body weight). In all, 841 patients were referred for ophthalmic assessment before or just after starting treatment with hydroxychloroquine (708 females, 133 males). The age range was 12-87 years and the distribution is shown in fig 1. Seventy six patients were referred for more than one course of hydroxychloroquine. A profile of the diseases treated is shown in the table. Most of the patients were examined by the author. Visual assessment included corrected visual acuity for distant and near vision, examination with a red Amsler grid, ${ }^{3-6}$ slit lamp examination, and funduscopy. Cumulative doses of hydroxychloroquine were calculated according to the patient's history of daily dose and duration.

\section{Results}

Following ophthalmic examination, 26 patients were felt to be unsuitable for treatment. Of these, 21 had existing macular abnormalities or pigmentary changes which were considered severe enough to make screening difficult. One patient had retinitis pigmentosa. In four, complete ocular examination was not possible because of cataract. A further 11 who had a satisfactory initial visual assessment were not started on treatment with hydroxychloroquine by the referring physician. Forty six of the patients referred failed to attend for initial ophthalmic assessment. During treatment with hydroxychloroquine ophthalmic examination was carried out at the eye clinic at regular six monthly intervals on 758 patients; 314 defaulted on at least one occasion.

\section{DOSE AND DURATION}

At the end of November 1995 it was possible to calculate the cumulative dose and duration of treatment with hydroxychloroquine in 713 cases. This included 495 patients who had stopped treatment, 108 who had defaulted from follow up, and 110 who were still receiving treatment (fig 2). The duration of treatment was up to one year in 302 patients $(42 \%)$, between one and two years in 176 $(25 \%)$, and between two and three years in 104 $(15 \%)$. Thirty five patients $(5 \%)$ had more than five years' treatment, the longest being 11 years. The starting dose was $400 \mathrm{mg} \mathrm{d}^{-1}$ in 611 cases. For those starting on $400 \mathrm{mg} \mathrm{d}^{-1}$, the rheumatologist reduced the dose during the treatment period in $84 \%$ of cases: $28 \%$ within six months, a further $32 \%$ within 12 months, and a further $24 \%$ within 18 months. In 272 patients $(38 \%)$ the cumulative dose of hydroxychloroquine was greater than $200 \mathrm{~g}$.
Accepted for publication 19 November 1996
Department of
Ophthalmology 16 Alexandra Parade United K31 2ER,

Correspondence to: 


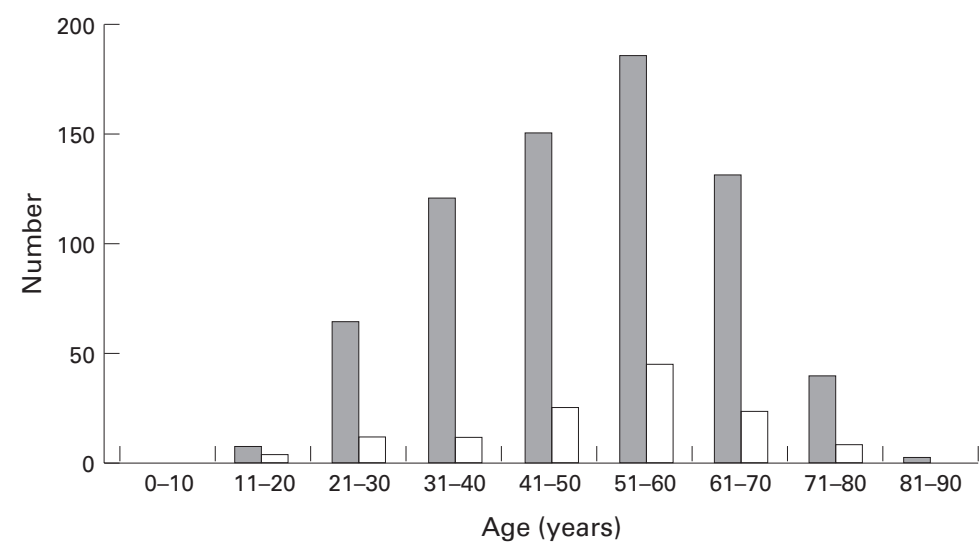

Figure 1 Age and sex distribution for patients on hydroxychloroquine; grey columns, female; white columns, male. tive dose 233.2 g. Over the next three years visual acuity and fundus appearance remained stable.

RETINAL PIGMENTATION

In addition to the patient described above, a further six patients were recorded as having pigmentary abnormalities at the posterior pole. Fluorescein angiography was carried out on all six. A small pigment epithelial window defect was reported in one. Four patients were reported to have age related macular changes. One patient, with a normal fluorescein angiogram, continued to take hydroxychloroquine and had a second course of treatment three years later. None of this group had any alteration in visual acuity at the time of assessment.

\section{CORNEAL CHANGES}

Corneal drug deposit was recorded in seven cases. ${ }^{4}$ One patient had also been on amiodarone for six months when the drug deposit was detected. Amiodarone is more likely to be the cause of the deposit in this instance. In four cases the daily dose of hydroxychloroquine was greater than $6.5 \mathrm{mg} \mathrm{kg}^{-1} \mathrm{~d}^{-1}$ and in one the cumulative dose was $514.6 \mathrm{~g}$. The cornea cleared either after stopping treatment or reducing the daily dose. All were asymptomatic.

\section{AMSLER GRID ABNORMALITIES}

Amsler grid changes were reported by 10 patients (shimmering, fuzzy, distorted). None of them had visual symptoms or alteration in visual acuity. No fundus or fluorescein angiogram abnormalities were detected in five, and retesting with an Amsler grid was normal. Of the remainder, two were myopic with pigmentary changes at the posterior pole, and two had age related macular changes. One 48 year old female patient reported focal areas of shimmering on the Amsler grid. Funduscopy revealed discrete areas of hypopigmentation around the left macula. These areas corresponded to retinal pigment epithelial window defects on fluorescein angiography. No central visual field defect was detected on testing with an automated visual field analyser. The appearance was not typical of chloroquine retinopathy, but treatment with hydroxychloroquine was stopped. She had received $400 \mathrm{mg}$ $\mathrm{d}^{-1}$ for about 17 months. The daily dose was estimated to be $7.4 \mathrm{mg} \mathrm{kg}^{-1} \mathrm{~d}^{-1}$ and the cumula-

Table 1 Disease profile of patients on hydroxychloroquine

\begin{tabular}{lrr}
\hline Disease & Female & Male \\
\hline Rheumatoid arthritis & 489 & 97 \\
Systemic lupus erythematosus & 118 & 5 \\
Mixed connective tissue disease & 27 & 5 \\
Seronegative polyarthritis & 25 & 2 \\
Juvenile chronic arthritis & 6 & 3 \\
Adult Still's disease & 0 & 2 \\
Discoid lupus erythematosus & 25 & 11 \\
Sarcoid & 2 & 0 \\
Benign vasculitis & 0 & 1 \\
Sjogren syndrome & 1 & 1 \\
Psoriatic arthropathy & 0 & 1 \\
Not recorded & 15 & 5 \\
\hline
\end{tabular}

VISUAL DISTURBANCE

Eight patients complained of visual disturbance. In three this was related to their general medical condition. One patient with SLE had a retinal vasculitis. In another who was anaemic and had recently undergone hip surgery there were signs of retinal ischaemia. The third had symptoms of amaurosis fugax. A further two patients made their own decision to stop treatment because they felt that their vision was blurred; one stopping after two days treatment. Ophthalmic examination was normal in both cases. Of the remainder, one patient Another complained of seeing red circles. In both cases the symptoms resolved after treatment was stopped. No ocular abnormality was detected in the former, and fundoscopy and fluorescein angiography revealed age related macular change in the latter. The final patient complained of blurred vision for close work but was not using her presbyopic spectacle correction. Although not specifically recorded, several patients had symptoms related to accommodation.

Four patients had problems with ocular muscle balance while on treatment with hydroxychloroquine. One patient with cerebral lupus complained of transient double vision. This resolved spontaneously and no ocular muscle imbalance was found when she was examined in the clinic. She continues on hydroxychloroquine. The second patient with SLE had difficulty with near vision and was found to have a superior oblique paresis. Of the remaining two, one complained of intermittent diplopia and the other of intermittent blurring of vision. The former was found to have a latent horizontal and vertical muscle imbalance, the latter had a long standing vertical muscle weakness. In both cases hydroxychloroquine was stopped. Unfortunately both patients failed to return for review.

\section{Discussion}

The reported incidence of retinal toxicity is low. ${ }^{24-12}$ Morsman et al summarise the incidence reported in other publications in a helpful table. ${ }^{6}$ Bernstein, ${ }^{5}$ reviewing the world literature, did not find hydroxychloroquine retinopathy where the dose did not exceed 6.5 complained of photophobia and lachrymation. 


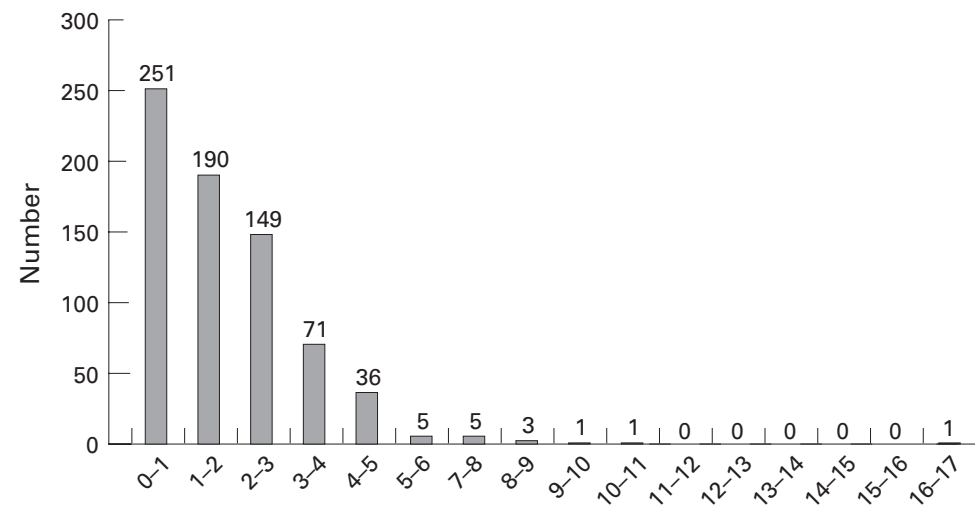

Hydroxychloroquine $(\mathrm{g} \times 100)$

Figure 2 Distribution of calculated cumulative dose of hydroxychloroquine at November 1995. Totals at top of column.

$\mathrm{mg} \mathrm{kg}^{-1}$ for more than 10 years. Many of the patients in this group did not remain on the standard daily dose of $400 \mathrm{mg}$ throughout the treatment period. The lower doses employed may have contributed to the avoidance of toxicity. Only one of our patients had a marked fundus abnormality. This was predominantly unilateral and not typical of chloroquine retinotoxicity. For those presenting with visual symptoms, there were no ophthalmic findings directly attributable to hydroxychloroquine. In the cases with ocular muscle imbalance there were insufficient data to draw any firm conclusions. A few of the older patients were noted to have age related macular changes but there was no evidence to suggest that hydroxychloroquine had a direct effect on these age related changes. The value of a baseline ophthalmic examination is underlined by the presence of significant pigmentary change around the macula in 21 patients. Bilateral pigmentary changes can occur in the absence of treatment with antimalarials, ${ }^{1314}$ age related macular changes being likely to occur in the older age group.

These data add to the existing pool of experience with hydroxychloroquine and suggest a low incidence of toxicity at the doses employed. In view of this, and since $82 \%$ of our patients received less than three years' treatment, we have adopted the College of Ophthalmologist guidelines. ${ }^{1}$ A baseline ophthalmic examination is performed before or soon after starting hydroxychloroquine. The patients are then given Amsler charts to use at home once a month. They are instructed to report any abnormalities immediately and stop treatment. At a daily dose of $400 \mathrm{mg} \mathrm{d}^{-1}$ a cumulative dose of $200 \mathrm{~g}$ will be reached after 18 months of treatment. The duration of treatment can, of course, be extended by reducing the daily dose. Many patients, however, derive symptomatic benefit from treatment with hydroxychloroquine and for some this benefit is such that they are prepared to continue treatment for several years. Those patients requiring treatment beyond the $200 \mathrm{~g}$ total are re-referred by the physician for ophthalmic assessment. The Amsler grid, although subjective, is a sensitive ${ }^{35}$ and easily administered test but can be affected by refractive errors and opacities in the ocular media. Nevertheless, in the absence of an ideal test for retinotoxicity, the Amsler grid remains an invaluable screening tool and offers a potential test for patients to use at home. The reliability of the self use of the Amsler grid at home remains to be fully assessed. Carmichael gives a useful guide for using hydroxychloroquine ${ }^{15}$ but the situation with regard to monitoring patients on longer term treatment still has to be addressed. This is tackled by Spalton et al. ${ }^{12}$ They recommend that, using a daily dose of $\leq 6.54 \mathrm{mg} \mathrm{kg}^{-1}$, all patients have an initial ophthalmic check and those having less than three years' treatment do not require further review. For patients on long term treatment he suggests annual check of acuity and fundoscopy between 5 and 10 years of treatment duration, with closer ophthalmic supervision for those requiring more than 10 years treatment.

I would like to thank Professor R D Sturrock for his advice and encouragement in preparing this review.

1 College of Ophthalmologists. Chloroquine, hydroxychloroquine and the eye. London: College of Ophthalmologists: 1993.

2 Mackenzie AH. Dose refinements in long-term therapy of rheumatoid arthritis with antimalarials. Am J Med 1983;75 (suppl):40-5.

3 Easterbrook M. The sensitivity of Amsler grid testing in early chloroquine retinopathy. Trans Ophthalmol Soc UK 1985;104:204-7

4 Easterbrook M. Ocular effects and safety of antimalarial agents. Am J Med 1988;85(suppl):23-9.

5 Bernstein HN. Ocular safety of hydroxychloroquine sulfate (Plaquenil). South Med J 1992;85:274-9.

6 Morsman CDG, Livesey SJ, Richards IM, Jessop JD, Mills PV. Screening for hydroxychloroquine retinal toxicity: Is It

7 Mills PV, Beck M, Power BJ. Assessment of the retinal toxicity of hydroxychloroquine. Trans Ophthalmol Soc UK icity of hydroxych

8 Tobin DR, Krohel GB, Rynes RI. Hydroxychloroquine. Seven-year experience. Arch Ophthalmol 1982;100:81-3. 9 Finbloom DS, Silver K, Newsome DA, Gunkel R. Comparison of hydroxychloroquine and chloroquine use and the development of retinal toxicity. J Rheumatol 1985; 12:692-4.

10 Mantyjarvi M. Hydroxychloroquine treatment and the eye. Scand J Rheumatol 1985;14:171-4

11 Johnson MW, Vine AK. Hydroxychloroquine therapy in massive total doses without retinal toxicity. Am J Ophthalmol 1987;104:139-144.

12 Spalton DJ, Verdon Roe GM, Hughes GRV. Hydroxychloroquine, dosage parameters and retinopathy. Lupus 1993; 2:355-8.

13 Scherbel AL, Mackenzie AH, Nousek JE, Atdjian M. Ocular lesions in rheumatoid arthritis and related disorders lar lesions in rheumatoid arthritis and related disorders with particular

14 Weise E, Yannuzzi LA. Ring maculopathies mimicking chloroquine retinopathy. Am J Ophthalmol 1974;78:204-10. 15 Carmichael AJ. Hydroxychloroquine: a guide to usage. J Dermatol Treat 1992;3:103-6. 